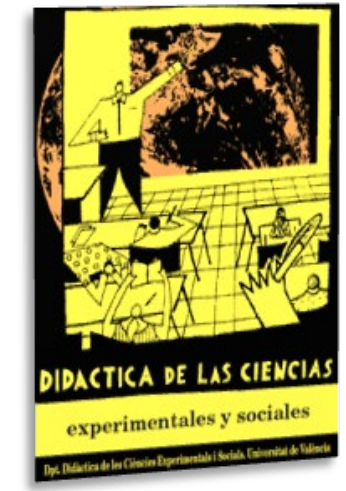

\title{
¿Qué aprenden unos estudiantes en una experiencia formativa de Didáctica de las Ciencias Experimentales en el Grado de Educación Infantil?
}

\author{
What do some students learn in a formative \\ experience of Teaching of Experimental \\ Sciences in Early Childhood Education?
}

DOI: $10.7203 / D C E S .38 .16174$

\author{
Carlos de Pro Chereguini \\ Universidadde Murcia, cpro@um.es \\ ORCID iD: https://orcid.org/0000-0002-4148-2910 \\ Carmen Inglés Carrillo \\ ISEN (Universidad de Murcia), carmen.inglesc@um.es \\ ORCID iD: https://orcid.org/0000-0002-5158-1066 \\ Alberto Gómez Mármol \\ Universidad de Murcia, alberto.gomez1@um.es \\ ORCID iD: https://orcid.org/0000-0002-6567-5012
}

\begin{abstract}
RESUMEN: Esta investigación pretende conocer si evolucionan los conocimientos iniciales de unas maestras de Educación Infantil en su formación inicial en el ámbito de la didáctica de las ciencias. La experiencia se realizó con estudiantes de $3^{\circ}$ del Grado de Educación Infantil, tras trabajar la propuesta "Cómo enseñar el cuerpo humano y los hábitos saludables en Educación Infantil”. Se utilizó un diseño cuantitativo no experimental con pretest y postest. Los resultados ponen de manifiesto los logros conseguidos, pero también revelan, de modo preocupante, las carencias con la que llegan y la insuficiente evolución tras esta primera intervención. Esto supone una llamada de atención para plantearnos qué podemos mejorar de su formación inicial.
\end{abstract}

Palabras Clave: formación inicial de maestros, enseñanza de las ciencias, Educación Infantil, conocimientos científicos y didácticos, cuerpo humano y salud.

ABSTRACT: This research aims to find out if the initial knowledge of Early Childhood Education teachers in their initial training in science teaching evolves. The experience was carried out with students in the 3rd year of the Degree in Early Childhood Education, after working on the proposal "How to teach the human body and healthy habits in Early Childhood Education. We have used a non-experimental quantitative design with pretest and posttest. The results show the achievements, but also reveal, worryingly, the shortcomings with which teachers present and the insufficient evolution after this first intervention. This is a wake-up call to ask what we can do to improve their initial training.

KEYWORDS: initial teacher training, science education, Early Childhood Education, scientific and teaching knowledge, human body and healthy habits.

Fecha de recepción: noviembre de 2019

Fecha de aceptación: junio de 2020 


\section{Problemas de inVestigación}

Algunos piensan que las necesidades formativas de una maestra de Educación Infantil (en adelante, EI) en el ámbito de la Didáctica de las Ciencias Experimentales (en adelante, DCE) son menores que en otros niveles, porque los conocimientos científicos que se deben enseñar son más elementales. Es la consecuencia de un principio arraigado: poner el acento en el contenido a enseñar y no en quién debe aprenderlo. En EI, el alumnado tiene de 0-6 años y, en consecuencia, presenta limitaciones comunicativas, un desarrollo cognitivo incompleto, un egocentrismo intelectual y social, formas singulares de aprender, etc. Minusvalorar, en estas circunstancias, la formación que precisa una maestra de esta etapa educativa en DCE, es sencillamente desconocer la realidad de la EI.

Creemos que es preciso tener referentes en los que articular un modelo formativo y concretar propuestas para las diferentes etapas del desarrollo profesional de los docentes. Para ello, deberíamos ser capaces de responder, entre otros, a una serie de interrogantes:

a) Qué pretende la EI del siglo XXI: ¿Qué las niñas y los niños aprendan sólo a leer y escribir? ¿Entretener mientras sus padres trabajan (una estrategia de conciliación familiar)? ¿Facilitar el proceso de socialización más allá de la familia? ¿Favorecer el desarrollo personal en todas sus facetas?... Obviamente la respuesta a estas cuestiones debe condicionar las siguientes.

b) Qué maestra necesitamos para ello: ¿Una docente con grandes conocimientos disciplinares? ¿Una experta con recursos (idiomas, telemáticos, artísticos...)? ¿Una profesional crítica y autocrítica, reflexiva, investigadora de su propia acción educativa? ¿Una persona afectiva con el alumnado? En definitiva, qué competencias debería adquirir una maestra para una escuela del siglo XXI.

c) Cómo articulamos la adquisición de estas competencias profesionales. Cualquier proceso formativo precisa, además de identificar las competencias deseables, una secuenciación de acciones y actividades que permiten evolucionar y construir los conocimientos (científicos, didácticos, prácticos, ideológicos, personales...) a lo largo de su desarrollo profesional. ¿Cuáles debemos afrontar en la etapa de formación inicial?

Pensamos que, quizás, no se han debatido suficientemente estas cuestiones en nuestra comunidad educativa y, desde luego, los resultados del hipotético debate no parecen haber presidido la elaboración de los planes de estudios. Probablemente esto explique que, a pesar de tener directrices comunes, haya tanta heterogeneidad en las asignaturas de DCE que impartimos. De hecho, si comparamos las Guías Docentes, encontramos diferencias en el número de materias y de créditos, en las prioridades formativas, en las competencias a desarrollar, en los contenidos... Estas disparidades no sólo provienen de las características de los formadores, lo que tendría una cierta justificación, sino de razones más profundas: ¿qué creemos los formadores que necesita saber, saber hacer, saber hacer con otros... una maestra de EI, en el ámbito de la DCE, en su etapa de formación inicial?

\section{LA FORMACIÓN INICIAL DE MAESTROS PARA ENSEÑAR CIENCIAS}

La formación del profesorado es un campo prioritario en la investigación en DCE; no obstante, en nuestro contexto educativo, el número de aportaciones es reducido si nos referimos a la formación inicial en EI. Algunas de ellas, agrupadas en ámbitos, las destacamos a continuación.

Se han realizado reflexiones sobre tópicos habituales de la DCE aplicados a la formación de maestras: la naturaleza de la ciencia (Acevedo, 2010); la argumentación (Archila, 2012); la visión de la enseñanza de las ciencias desde el constructivismo (Marín, 2014); las prácticas de carácter científico (Bargiela, Puig y Blanco, 2018). Otras se han ocupado de las dificultades que presenta la formación inicial (Pro Bueno, 2016). 
Hay trabajos centrados en la identificación de los conocimientos de las futuras maestras: sobre contenidos disciplinares (Bravo y Cañada, 2016; Ferrer, De Echave y Mateo, 2016); sobre contenidos didácticos (Valín, Moledo, López-Maceiras y García, 2012; Arias, Álvarez y Álvarez, 2013; Lorca, Vázquez y Rosa, 2014; López-Banet y Pro, 2016); y, en menor medida, sobre la experiencia y práctica profesional (Cantó, Pro Bueno y Solbes, 2016; Mazas y Bravo, 2016). También hay contribuciones sobre los intereses de los futuros maestros (Cantó y Solbes, 2014) o sobre los resultados obtenidos en DCE (Pablos y Verde, 2013).

Se plantean propuestas o actividades concretas sin resultados empíricos para trabajar en la formación inicial. Entre ellas, encontramos unas más orientadas a la actualización disciplinar (Sánchez, Conde y Garrido, 2014; Romero-López, Jiménez-Tejada, González-García, CarrilloRosúa, Barón, Casas-Castillo y Ruiz-Rodríguez, 2016) y otras con un enfoque más didácticoprofesional (Do Carmo, Jiménez-Liso, López, Porlán y Rivero, 2016; Sanz y Gutiérrez, 2017).

También encontramos estudios sobre la evolución histórica de la DCE (Jiménez, 2012) y análisis de trabajos en congresos (Solé, Aguilar, Ibáñez y Coiduras, 2017; 2018).

Por último, como nuestro trabajo se refiere a la puesta en práctica y la evaluación de una propuesta, hemos sintetizado algunas aportaciones que se recogen en la Tabla 1.

TABLA 1. Aportaciones sobre propuestas para enseñar ciencias en EI

\begin{tabular}{llc}
\hline \multicolumn{1}{c}{ AUTORES } & \multicolumn{1}{c}{ TEMÁTICA DE LA INVESTIGACIÓN } & TIPO \\
\hline Aragüés y Sáez (2014) & $\begin{array}{l}\text { Análisis de una experiencia sobre el modelo de reacción } \\
\text { química y de ósmosis con los futuros maestros de EI }\end{array}$ & IPC \\
\hline Calafell y Banqué (2017) & $\begin{array}{l}\text { Análisis y evaluación para ambientalizar la sostenibilidad en } \\
\text { la formación de maestros de EI }\end{array}$ & IPC \\
\hline Cantó, Pro y Solbes (2017) & $\begin{array}{l}\text { Conocimientos de los futuros maestros de EI al elaborar } \\
\text { unidades didácticas de ciencias }\end{array}$ & IPD \\
\hline Díaz (2017) & Análisis de propuestas para trabajar la indagación en EI & IPD \\
\hline $\begin{array}{l}\text { Eugenio, Aragón, Jiménez y } \\
\text { Vicente (2016) }\end{array}$ & $\begin{array}{l}\text { Análisis de una propuesta didáctica para alfabetizar } \\
\text { científicamente a los futuros maestros de EI }\end{array}$ & IPC \\
\hline $\begin{array}{l}\text { López-Luengo, Gil, Verde y } \\
\text { Vallés (2012) }\end{array}$ & $\begin{array}{l}\text { Análisis de la metodología y evaluación de una propuesta de } \\
\text { enseñanza-aprendizaje de las ciencias para el Grado de EI }\end{array}$ & IPD \\
\hline $\begin{array}{l}\text { López-Luengo, Vallés y Gil } \\
\text { (2016) }\end{array}$ & $\begin{array}{l}\text { Análisis de una propuesta didáctico-científica para alumnos } \\
\text { del Grado de EI }\end{array}$ & IPD \\
\hline $\begin{array}{l}\text { Moreno, González y Meneses } \\
\text { (2017) }\end{array}$ & $\begin{array}{l}\text { Uso de una metodología de indagación en EI: “Limpiemos el } \\
\text { agua" }\end{array}$ & IPD \\
\hline Pro Chereguini (2017a) & $\begin{array}{l}\text { Conocimientos didácticos de los futuros maestros de EI para } \\
\text { evaluar hábitos saludables }\end{array}$ & IPD \\
\hline Pro Chereguini (2017b) & $\begin{array}{l}\text { Análisis de propuestas de los futuros maestros de EI para } \\
\text { trabajar actividades experimentales }\end{array}$ & IPD \\
\hline Sanz y Gutiérrez (2016) & $\begin{array}{l}\text { Desarrollo de un modelo de investigación-acción con talleres } \\
\text { científicos para escolares y profesorado de EI }\end{array}$ & IPD \\
\hline $\begin{array}{l}\text { Vestena, Pretto y Marschall } \\
\text { (2017) }\end{array}$ & $\begin{array}{l}\text { Análisis de una propuesta de trabajo supervisado sobre las } \\
\text { ciencias en EI }\end{array}$ & IPC \\
\hline
\end{tabular}

IPC: investigación propuesta científica IPD: investigación propuesta didáctica

Fuente: elaboración propia 
Un análisis de las contribuciones señaladas pone de manifiesto, entre otras:

a) la insuficiencia de las investigaciones sobre propuestas para la enseñanza de la DCE en la formación inicial de las maestras de esta etapa;

b) la importancia de conocer y evaluar lo que estamos haciendo en las asignaturas que impartimos, no sólo por las carencias investigadoras ya mencionadas sino por tener datos y reflexionar sobre la labor formativa que estamos realizando;

c) la creencia de que la finalidad de nuestras asignaturas es actualizar científicamente a las futuras maestras (repetir formación), amparados en las carencias iniciales en dichos conocimientos. En este sentido, algunos especialistas (entre otros, Díaz, 2017; Solé, Aguilar, Ibáñez y Coiduras, 2017; Bargiela et al., 2018; etc.) admiten que se podrían conseguir estudiantes con unos conocimientos científicos más sólidos, pero no sabrían cómo trabajarlos en las aulas de EI. Como apuntan Cantó et al. (2017), no es lo mismo enseñar contenidos científicos a estudiantes de secundaria que a futuros docentes de EI; simplemente porque ambos colectivos tienen distintas necesidades formativas.

d) la obligación permanente de revisar y debatir qué podemos aportar desde la DCE para atender las necesidades de las maestras y cómo hacerlo (Do Carmo et al., 2016).

\section{Planteamiento del PROBlema de la inVestigación}

Además de las aportaciones de la innovación e investigación educativas, hay que considerar otros factores como el currículum oficial, no para asumirlo sino para comprender su alcance. De hecho, el Real Decreto 1630/2006, que establece las enseñanzas mínimas del $2^{\circ}$ ciclo de EI (MEC, 2014), contempla: conocer su cuerpo y el de los otros; observar, explorar y valorar su entorno natural; adquirir autonomía en la higiene y alimentación; desarrollar sus capacidades afectivas y comunicativas; construir su propia identidad, etc. Es decir, hay que facilitar pautas para que la futura maestra sea capaz enseñar estos conocimientos.

Por ello y compartiendo lo señalado por Do Carmo et al. (2016), vamos a reflexionar sobre nuestra propia práctica docente. Por ello, nuestro interrogante central es: ¿Qué efectos produce la propuesta "Cómo enseñar el cuerpo humano y los hábitos saludables en EI" en el alumnado del Grado de Educación Infantil?

Para organizar el estudio, lo desdoblamos en dos problemas:

- Problema Principal 1 (PP1). ¿Cómo utilizaban sus conocimientos sobre "el cuerpo humano y la salud" las futuras maestras de EI, antes de trabajar la propuesta? ¿De qué dependían los resultados?

- Problema Principal 2 (PP2). ¿Cómo utilizaban sus conocimientos las futuras maestras, tras cursar la propuesta “Cómo enseñar el cuerpo humano y los hábitos saludables en EI”? ¿Se han producido progresos significativos en dichos conocimientos?

\section{Metodología de LA INVESTigación}

Son muchas las características que definen a una maestra: conocimientos científicos y didácticos, experiencia profesional, cualidades personales o creencias ideológicas (Cantó et al., 2017). En definitiva, variables complejas que imposibilitan estudiarlas a la vez y con los mismos alumnos. En este trabajo solo nos ocupamos de algunas: conocimientos científicos (CC) y los conocimientos didácticos (CD) de unas maestras en su formación inicial.

El diseño que hemos utilizado es un estudio longitudinal con un pretest antes de nuestra intervención y un postest tras la misma. La principal característica de nuestra propuesta es que se trata de la primera asignatura de DCE que cursan las estudiantes de la titulación de Grado de EI. 


\subsection{Participantes}

Respecto a los participantes, se seleccionaron por su disponibilidad, cercanía, implicación... Eran un total de 42 alumnos (41 alumnas y 1 alumno) matriculados en "Enseñanza y Aprendizaje de las Ciencias de la Naturaleza I" (EACN1); no obstante, solo disponemos de los datos de 34 alumnas, todas mujeres.

Las variables respecto a las que vamos a estudiar la dependencia son:

-Edad. La edad media de los participantes era 22 años; el 76\% oscilaba entre 20 y 22 años, "edad habitual" en $3^{\circ}$ de un Grado.

- Valoración de la enseñanza de las Ciencias en EI. Mayoritariamente, aludían a múltiples razones: su importancia en la vida cotidiana, su necesidad para conocer el entorno, su interés por conocerlas, su afinidad escolar frente a otras materias... No obstante, también los había que mostraban un cierto rechazo. Globalmente podemos decir que 24 alumnas tenían una opinión positiva $(70 \%), 4$ una neutra $(12 \%)$ y 6 una negativa $(18 \%)$.

- Estudios de procedencia. En cuanto a los estudios anteriores a sus entradas en la universidad, sólo 5 habían cursado el Bachillerato de Ciencias (15\%); los demás habían realizado 20 el de Sociales (59\%); 8 el de Humanidades (23\%) y 1 el de Artes (3\%).

- Tiempo desde que cursaron Ciencias. Solo 3 habían cursado materias científicas en los últimos tres años $(9 \%)$.

\subsection{Contexto}

Como hemos dicho, la experiencia se realizó en la asignatura EACN1, primera de DCE en el plan de estudios del Grado de EI. Se trata de una materia de $3^{\circ}$, obligatoria y presencial, desarrollada en el $2^{\circ}$ cuatrimestre en la Universidad de Murcia. Tenía 6 créditos ECTS, lo que suponía un volumen de trabajo -del estudiante- de 150 horas, repartidas en horas presenciales (sesiones teórico-prácticas con el grupo clase, desdobles para las actividades prácticas, tutorías y examen escrito) y horas de trabajo autónomo. La materia se impartió durante 7 horas semanales, durante 8 semanas.

\section{Datos sobre nuestra propuesta de enseñanza}

El tema de las competencias generales, trasversales, de las materias... se ha convertido muchas veces en "formalidades" que poco o nada tienen que ver con "lo real". Sin embargo, creemos que -más allá de las exigencias administrativas- pueden ser un elemento curricular importante en los títulos profesionales. Entre las competencias "reales" que trabajamos estaban:

- Conocer los objetivos, contenidos y criterios de evaluación de Ciencias en EI y debatir las razones para enseñar Ciencias en la EI.

- Valorar la importancia de la igualdad, la solidaridad, la diversidad, la equidad y el respeto a los derechos humanos como factores a considerar en la enseñanza de las Ciencias en EI.

- Justificar qué deberían saber, saber hacer, saber hacer con otros, saber ser y estar... en EI (en ambos ciclos) en relación con el cuerpo humano y la creación de hábitos saludables.

- Identificar características y experiencias de los estudiantes que condicionan o facilitan la adquisición de conocimientos sobre el cuerpo humano y la creación de hábitos saludables.

- Aprender estrategias para fomentar un clima lúdico e inclusivo en las clases de ciencias. Conocer secuencias de actividades para enseñar ciencias en EI.

- Analizar propuestas sobre el cuerpo humano y la salud.

- Analizar materiales y recursos para enseñar el cuerpo humano y la salud.

- Diseñar materiales para enseñar conocimientos concretos (sobre obesidad, higiene, alimentación...). 
- Analizar instrumentos de evaluación del aprendizaje, identificando intencionalidad y características.

En cuanto a los contenidos, la materia se estructuraba en seis temas donde se trabajaban indistintamente conocimientos científicos y didácticos. La Imagen 1 esquematiza esta información.

IMAGEN 1. Conocimientos científicos y didácticos de la asignatura EACN1

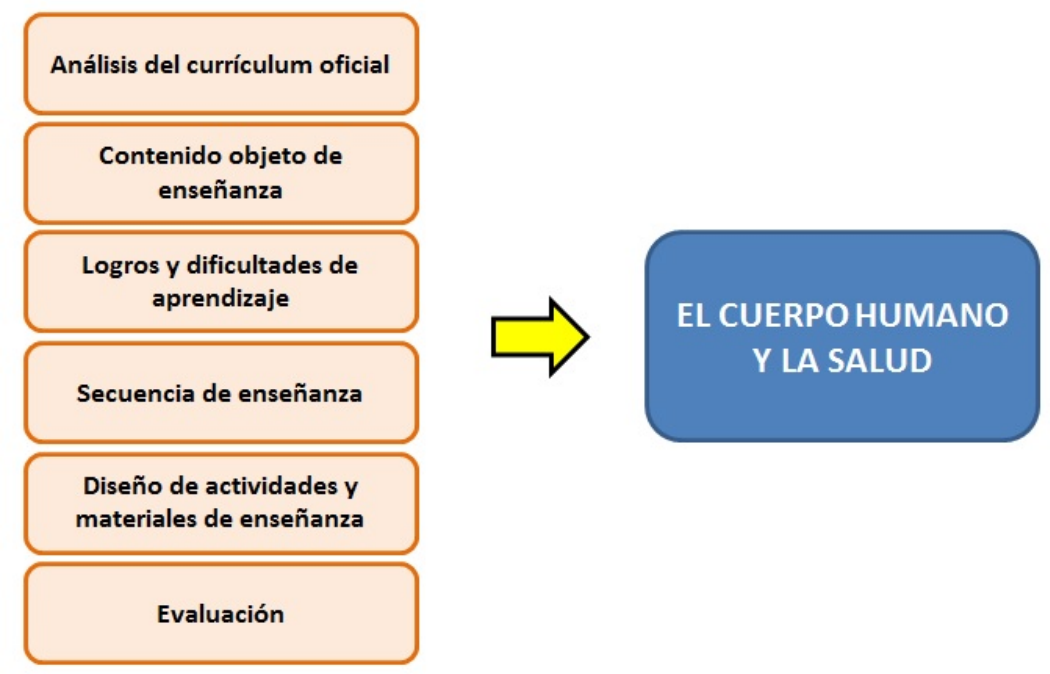

Fuente: elaboración propia

Para trabajar estos contenidos, el formador planteó la secuencia recogida en el Anexo 1.

\subsection{Instrumentos de recogida de información}

Queríamos conocer los conocimientos -científicos (en adelante, CC) y didácticos (en adelante, CD) - de nuestros estudiantes para enseñar "el cuerpo humano y la salud" en EI, antes (pretest) y después (postest) de cursar EACN1.

Para aportar respuestas al PP1, el primer día de clase se pasó el pretest sin límite de tiempo. Tenía 19 cuestiones; 17 se ocupaban del CC inicial de las alumnas. No indagamos mucho en el CD ya que no habían cursado ninguna asignatura de DCE; no obstante, incluimos dos cuestiones de didáctica general que se habían cursado para que las aplicaran al ámbito de las ciencias. El cuestionario completo se recoge en http://territoriopro.com/documentos/EACN1_pretest.pdf.

Partiendo de las respuestas en el pretest, categorizamos los CC y los CD del alumnado según su porcentaje de acierto en los ítems de la prueba. Luego, estudiamos la dependencia con algunas variables: edad, visión de las Ciencias en EI, modalidad de acceso y tiempo desde la última vez que tuvieron relación con las ciencias. Para ello, calculamos el coeficiente $\rho$ de Spearman y la $\chi^{2}$ de Pearson.

En relación con el PP2, se pasó un postest. Fueron preguntas abiertas, semejantes al pretest, y se realizó el último día de clase. Duró dos horas y constaba de 15 preguntas, de CC y CD, recogidos en http://territoriopro.com/documentos/EACN1_postest.pdf.

También categorizamos los CC y los CD del postest, según su porcentaje de acierto en los ítems de la prueba, tal y como habíamos hecho con el pretest. Con ello pudimos valorar la significación de las diferencias entre el pretest y el postest, utilizamos las tasas de acierto (CC y $\mathrm{CD})$ de las alumnas en ambas pruebas y aplicamos la prueba $\mathrm{W}$ de Wilcoxon. Por último, estudiamos la relación entre pretest y postest mediante la $\rho$ de Spearman. 


\section{ANÁLISIS E INTERPRETACIÓN DE LOS RESULTADOS}

En un trabajo, no podemos valorar todos los conocimientos adquiridos ni pretendemos generalizar las conclusiones en una experiencia tan contextualizada. No obstante, vamos a describir algunos resultados, manteniendo la diferenciación por problemas.

\subsection{Análisis e interpretación de los resultados del Problema Principal 1 (PP1)}

El PP1 se centraba en cómo utilizaban sus conocimientos sobre "el cuerpo humano y la salud" las futuras maestras de EI, antes de comenzar la propuesta "Cómo enseñar el cuerpo humano y los hábitos saludables en EI” y de qué dependían los resultados.

\section{¿Cómo utilizaban sus conocimientos científicos (pretest)?}

En la Tabla 2 se recoge el porcentaje de acierto del CC en el pretest.

TABLA 2. Tasa de aciertos en el pretest del CC

\begin{tabular}{lc}
\hline \multicolumn{1}{l}{ DESCRIPCIÓN DE CUESTIONES } & TASA DE \\
& ACIERTO \\
\hline ¿Por qué estornudamos? & $21 \%$ \\
\hline Recorrido de la sangre & $0 \%$ \\
\hline ¿Cómo se producen las cosquillas y por qué no podemos hacérnoslas nosotros? & $6 \%$ \\
\hline Ejemplificar un desayuno equilibrado para niños & $53 \%$ \\
\hline Indicar la distribución porcentual diaria de macronutrientes que deben consumir los niños & $0 \%$ \\
\hline Indicar los alimentos principales que debe contener una dieta equilibrada para niños & $29 \%$ \\
\hline Dada la información nutricional de dos productos, justificar cuál nos haría "engordar” más & $65 \%$ \\
\hline $\begin{array}{l}\text { Dada la información nutricional de dos productos, justificar cuál sería mejor para el } \\
\text { crecimiento y la renovación de tejidos }\end{array}$ & $18 \%$ \\
\hline Dada la información nutricional de dos productos, justificar cuál sería mejor para la piel & $0 \%$ \\
\hline Justificar cuándo es mejor lavarse los dientes (desayuno, comida o cena) & $26 \%$ \\
\hline Justificar la importancia de dormir un mínimo de horas al día para los niños & $9 \%$ \\
\hline Indicar 5 hábitos relacionados con la higiene infantil & $29 \%$ \\
\hline \begin{tabular}{l} 
Justificar acuerdo/desacuerdo de: "La respiración solo tiene lugar en los pulmones” \\
\hline Justificar acuerdo/desacuerdo de: "El órgano principal en la digestión es el estómago"
\end{tabular} & $0 \%$ \\
\hline $\begin{array}{l}\text { Justificar acuerdo/desacuerdo de: "Las arterias siempre llevan la sangre desde el corazón al } \\
\text { resto de partes del cuerpo y las venas siempre lo hacen desde los capilares al corazón” }\end{array}$ & $3 \%$ \\
\hline $\begin{array}{l}\text { Justificar acuerdo/desacuerdo de: "Es recomendable lavarse los dientes siempre justo } \\
\text { después de comer" }\end{array}$ & $0 \%$ \\
\hline $\begin{array}{l}\text { Justificar acuerdo/desacuerdo de: "Las vitaminas son nutrientes energéticos que actúan en } \\
\text { todos los procesos fisiológicos” }\end{array}$ & $6 \%$ \\
\hline
\end{tabular}

Fuente: elaboración propia

Una síntesis de los resultados en los que subyacían los CC se recoge a continuación.

- Por qué estornudamos: hubo tres argumentos que consideramos adecuados: mecanismo de defensa del aparato respiratorio, respuesta a una alergia o consecuencia de un problema medioambiental. Sólo siete alumnos mencionaron dos de ellos.

- Recorrido de la sangre por el cuerpo: ninguno lo describió adecuadamente; sólo una (A8) aludió a la circulación sistémica y la pulmonar, sin mencionar mucho más. En general, dieron 
respuestas pobres y confusiones (por ejemplo, la funcionalidad de las venas y arterias, o de aurículas y ventrículos).

- Producción de cosquillas: sólo dos (A14 y A23) hablaron de zonas sensibles y que no podíamos hacérnoslas porque somos conscientes y el cuerpo se protege. Un porcentaje importante no respondió (24\%) o esgrimió razones inadecuadas $(72 \%)$, a pesar de ser una práctica habitual de muchos de los colegios de la Región de Murcia como actividad de relajación.

- Composición de un desayuno equilibrado: algo más de la mitad propuso uno completo o parcialmente equilibrado, combinando fruta (pieza o zumo), lácteos (leche o yogur) y cereales, tostadas o galletas. Quizás, en estos resultados más adecuados, influyera la sensibilización actual de los adolescentes por estas temáticas.

- Distribución diaria de hidratos de carbono, proteínas y grasas para los niños: ninguno respondió de forma deseable (hidratos de carbono 50-60\%; grasas 30-35\%; proteínas 10-15\%). La mitad no respondió y la otra no solo indicó una distribución inadecuada, sino que la suma de porcentajes no daba el $100 \%$ en muchos casos (44\%).

- Identificación de los alimentos que debe contener una dieta equilibrada: solo diez los identificaron adecuadamente. Hablaron de frutas (79\%), pescados $(62 \%)$, carnes $(59 \%)$, verduras (59\%) y mencionaron otros alimentos como lácteos $(32 \%)$, cereales $(24 \%)$ y legumbres $(18 \%)$. Las principales omisiones fueron verduras y lácteos.

- Información nutricional de las etiquetas de dos tipos de leche: un 65\% respondieron adecuadamente cuál engordaba más: "porque tiene más calorías" o "porque lleva más azúcar y grasas saturadas". En cuanto a cuál era mejor para el crecimiento, disminuyeron las respuestas deseables "porque tiene más proteínas" (18\%); asociaron la renovación de los tejidos a la presencia de minerales (38\%) o vitaminas (35\%). Y, en cuanto cuál era mejor para la piel, siendo la respuesta buscada "ninguna leche es mejor que la otra para la piel", no encontramos contestaciones adecuadas.

- Momento mejor para lavarse los dientes: casi la mitad indicaron "tras la cena", aunque sólo el 26\% lo justificaron "porque es el período más largo de inactividad entre ingestas de comida donde las bacterias pueden actuar". Hubo despistes porque, aunque solo debían elegir una, el 38\% respondieron: "las tres son importantes".

- Importancia de las horas de sueño deseables: consideramos adecuadas las que se referían a la mejora del rendimiento escolar, a favorecer la atención y el buen comportamiento, a la influencia en el metabolismo, el peso y la altura, a la regulación y reparación del organismo, etc. Sólo 3 alumnas dieron justificaciones acertadas; entre las erróneas, estarían "para tener activo el cerebro" (9\%) o "para asimilar nutrientes" (9\%).

- Hábitos de higiene: la media de hábitos correctos fue 3,8; lo que justifica que el $71 \%$ no lo hiciera acertadamente. Los hábitos más repetidos fueron "lavarse los dientes" (100\%), "lavarse las manos" (94\%), "ducharse/bañarse" (59\%) y "lavarse la cara” (32\%). Algunas (12\%) incluyeron hábitos que no son de higiene "comer saludablemente" o "dormir bien".

- Respecto al acuerdo o desacuerdo justificado sobre una serie de afirmaciones: encontramos que, en el caso de la respiración, ninguno aludió a la respiración celular. Un $21 \%$ indicó que era falsa la afirmación que el órgano principal de la digestión era el estómago, pero sólo A16 fue capaz de justificarlo. En cuanto a dónde llevan la sangre las arterias y las venas, casi un 40\% acertaron que no era correcta, pero ninguna lo justificó bien. En cuanto al momento idóneo para lavarse los dientes, un $26 \%$ acertó y solo dos (A13 y A26) lo justificaron. Y en relación con el hecho de que las vitaminas eran nutrientes energéticos, sólo un $26 \%$ dijo que era falsa, pero ninguna lo argumentó.

En resumen, el pretest de la asignatura EACN1 reflejó importantes carencias en cuanto al CC antes de iniciar su formación en DCE, principalmente en la utilización de los conocimientos sobre "el cuerpo humano"; parecen conocer más sobre "la alimentación", sin que tengamos claro si se debe a la escuela o a conocimientos realizados fuera de ella. 
¿Cómo utilizaban sus conocimientos didácticos (pretest)?

En la Tabla 3 se recoge el porcentaje de acierto del CD en el pretest.

TABLA 3. Tasa de aciertos en el pretest del CD

\begin{tabular}{lc}
\hline \multicolumn{1}{c}{ DESCRIPCIÓN DE CUESTIONES } & TASA DE \\
ACIERTO
\end{tabular}

Fuente: elaboración propia

Una síntesis de los resultados en los que subyacían los CD se recoge seguidamente.

Aunque las características de los alumnos que condicionen el aprendizaje se habían trabajado en la asignatura "Diversidad en el aprendizaje y el desarrollo infantil" de $2^{\circ}$ curso, nadie contestó adecuadamente cuando se les pidieron cinco de ellas. La media de aciertos fue 0,9 . Un $32 \%$ no respondió y hubo respuestas que no pedíamos: orientaciones metodológicas, hábitos, elementos del contexto y la familia. Entre las correctas, mencionamos "ritmo de aprendizaje" (12\%) y “capacidades psicomotoras" (9\%).

En cuanto a los principios metodológicos que habría que considerar para la enseñanza en EI se habían trabajado en varias asignaturas ya cursadas -en $1^{\circ}, 2^{\circ}$ y primer cuatrimestre de $3^{\circ}$ - sólo ocho alumnas respondieron adecuadamente (24\%). La media de aciertos fue 1.4 (47\%); las de mayor presencia fueron "manipulación y experimentación" (35\%), "el juego" (21\%), "partir de los conocimientos previos del alumnado" (15\%), "materiales o actividades motivadoras" (15\%) y "la interacción con el medio que les rodea" (12\%). Nuevamente hubo alumnas que no contestaron lo que preguntamos o no respondieron $(15 \%)$.

En resumen, también se observaron carencias en sus $\mathrm{CD}$ que habían trabajado en otras asignaturas del Grado y más recientemente que los CC; estas limitaciones, normalmente menos mencionadas, suponen un obstáculo añadido para la DCE.

¿De qué dependian los resultados del pretest?

La categorización de los CC y los CD del alumnado en el pretest se muestra en la Tabla 4.

TABLA 4. Categorización del CC y CD del alumnado en el pretest

\begin{tabular}{ccc}
\hline $\begin{array}{c}\text { CATEGORÍA } \\
(\text { CC) }\end{array}$ & $\begin{array}{c}\text { TASA DE } \\
\text { ACIERTO }\end{array}$ & ALUMNAS \\
\hline Nada aceptable & Menos del $50 \%$ & Todas \\
\hline $\begin{array}{c}\text { CATEGORÍA } \\
\text { (CD) }\end{array}$ & TASA DE & ALUMNAS \\
\hline ACIERTO & A1, A9, A14, A17, A22, A26, A27, A32 \\
\hline Nada aceptable & Entre 50-59\% & Restantes \\
\hline
\end{tabular}

Fuente: elaboración propia

En cuanto a la dependencia de los resultados del pretest con las variables mencionadas, calculamos el coeficiente de correlación correspondiente y obtuvimos: 
- No hay relación estadísticamente significativa entre CC (pretest) y la edad, la modalidad de acceso y la visión que tienen de las Ciencias en EI.

-Hay una relación significativa $(p<0,05)$ entre CC (pretest) y el tiempo trascurrido desde que estudiaron ciencias $(\rho=-0,37)$.

- No hay una relación estadísticamente significativa entre CD (pretest) y las variables estudiadas.

Por lo tanto, hemos encontrado relaciones "esperables" (la negativa con el tiempo desde que estudiaron Ciencias) y otras "no esperables" (no dependen de la modalidad de Bachillerato ni de la visión que tienen de las Ciencias).

En cualquier caso, el profesor de la materia debió adaptarse a las condiciones iniciales del alumnado sin obviar la guía docente de la asignatura.

\subsection{Análisis e interpretación de los resultados del Problema Principal 2 (PP2)}

El PP2 se centraba en cómo utilizaban sus conocimientos sobre "el cuerpo humano y la salud" las futuras maestras de EI, tras trabajar la propuesta "Cómo enseñar el cuerpo humano y los hábitos saludables en EI” y si se habían producido progresos significativos.

\section{¿Cómo utilizaban sus conocimientos científicos (postest)?}

En la Tabla 5 se recoge el porcentaje de acierto del CC en el postest.

TABLA 5. Tasa de aciertos en el postest del CC

\begin{tabular}{lc}
\hline \multicolumn{1}{c}{ DESCRIPCIÓN DE CUESTIONES } & TASA DE \\
ACIERTO
\end{tabular}

Fuente: elaboración propia

Una síntesis de los resultados en los que subyacían los CC se recoge a continuación.

- Valoración energética de un desayuno infantil: calculando las kilocalorías que aporta, debían concluir que aportaba más kilocalorías de las recomendables para un niño de 5 años. Además de los que realizaron el ejercicio correctamente, un $18 \%$ más lo justificó adecuadamente pese a errar en los cálculos (olvido de unidades, descuido en alguna operación, ...). Un $24 \%$ no lo justificó de 
forma coherente y el $29 \%$ cometió errores de cálculo, de nuevo problemas con la competencia matemática.

- Acciones a partir de la información nutricional de un desayuno: es una cuestión con mayor dificultad que en el pretest. No obstante, un 56\% indicó que el desayuno era saludable, ya que la cantidad de macronutrientes era la recomendada $\mathrm{y}$, en los pequeños desajustes, proponían compensarlo en las otras comidas del día. Por otra parte, creemos que las alumnas, que no justificaron o lo hicieron de forma incompleta, tenían dificultades al trabajar con porcentajes.

- Información nutricional de dos cereales: todas acertaron cuál engordaba más aunque casi la cuarta parte no lo justificó adecuadamente: "porque lleva más azúcares y más grasas saturadas". En cuanto cuál sería mejor para crecer y renovar tejidos, lo justificaron bien todas salvo A2 y A28, mejorando uno de los mejores resultados del pretest (56\%). Respecto a cuál sería mejor para el tránsito intestinal todas respondieron y lo justificaron acertadamente, excepto A26, "porque tiene más fibra".

- En relación con las frases similares que en el pretest: todas -salvo A4, A7 y A31demostraron haber asimilado el concepto de respiración y diferenciado la pulmonar y la celular. En cuanto al órgano principal de la digestión, no sólo respondieron adecuadamente, sino que lo justificaron. En cuanto a la función de las venas y las arterias en la circulación, sólo la mitad detectó que los papeles se habían intercambiado en la frase. Todos reconocieron que el proceso de excreción no se limita a la orina y lo justificaron adecuadamente salvo A7 y A10. Y, sobre el valor energético de las vitaminas y las sales, la mayoría reconoció que estos eran nutrientes no energéticos, aunque el $21 \%$ tuvieron dificultades para justificarlo; confundieron "procesos reguladores" y "procesos plásticos”, función propia de las proteínas.

\section{¿Cómo utilizaban sus conocimientos didácticos (postest)?}

En la Tabla 6 se recoge el porcentaje de acierto del CD en el postest.

TABLA 6. Tasa de aciertos en el postest del CD

\begin{tabular}{lc}
\hline \multicolumn{1}{c}{ DESCRIPCIÓN DE CUESTIONES } & TASA DE \\
ACIERTO
\end{tabular}

Fuente: elaboración propia

Una síntesis de los resultados en los que subyacían los CD se recoge seguidamente.

- Indicar 5 características de los alumnos que condicionen el aprendizaje de las ciencias en EI, todas respondieron el ítem con una tasa de acierto apreciablemente alta. Como anécdota, señalamos que la media de identificaciones correctas fue 5,4 características (por encima del valor máximo); varias estudiantes propusieron más de las pedidas. Las más escogidas fueron "motivación" (59\%), "déficit atencional" (50\%), "personalidad" (47\%), "ritmo de aprendizaje" (41\%) e "inadaptación social" (41\%).

- Orientaciones metodológicas para enseñar ciencias en EI: la mitad respondió correctamente. Con las mismas exigencias que el pretest -sólo tres orientaciones- el porcentaje subiría al 75\%, casi 
todos los que contestaron. No todo fue satisfactorio: un $25 \%$ no respondió. Las orientaciones más repetidas fueron "constructivismo" (62\%), "globalización" (59\%), "resolución infantil de problemas" (59\%), "aprendizaje significativo" (35\%), "juego" (12\%) e "interacción con el medio" $(12 \%)$, éstas dos últimas habían aparecido en el pretest.

- Planteamiento de una actividad para aplicar las fases del método científico: más de la mitad realizó bien el ejercicio. Entre los fallos (35\%) encontramos: no formulaban preguntas que generasen hipótesis, no registraban los resultados del experimento en un lugar visible para los niños, la profesora era la que interpretaba los resultados, no los relacionaban con las hipótesis o no aportaban conclusiones del experimento. Las temáticas más utilizadas fueron la "germinación de plantas" (26\%) y la "mezcla de colores" (21\%). Globalmente, consideramos buenos los resultados, pero mejorables.

- Identificación de la fase de la secuencia de una actividad: un $82 \%$ realizaron y justificaron el ejercicio adecuadamente (fase de construcción y de aplicación). De ellas, 15 indicaron las dos posibles fases válidas, 8 justificaron solo la "construcción del aprendizaje" y 5 solo la "aplicación". Las demás incluyeron otras fases (explicitación, orientación o revisión).

- Identificar los tipos de contenidos a trabajar con una actividad: hemos de indicar que era un texto extenso y que cabrían distintas interpretaciones, aunque había un núcleo incuestionable. La mitad identificó adecuadamente los conceptos, procedimientos y actitudes de la actividad. De las estudiantes que hicieron una identificación incompleta, al 50\% les faltaba algún procedimiento y al $35 \%$ alguna actitud; en ambos suelen tener dificultades de identificación por el alumnado. En el resto, el error más frecuente fue redactar algún contenido como objetivo (24\%) o confundir un contenido procedimental con uno actitudinal $(6 \%)$.

La media de los elegidos fue 11,8 contenidos (4,2 conceptuales; 4,6 procedimentales; y 3 actitudinales), cerca de los 13 que esperábamos. La mayoría de los conceptos escogidos fueron los esperados: "principios básicos del funcionamiento de la vista y la pupila" (68\%), "la visión y los efectos de la luz sobre el ojo" (62\%), "el ojo" (53\%) y "las funciones de contracción y dilatación de la pupila" (53\%). Respecto a los procedimentales destacaron: "observación de fenómenos: contracción y dilatación de la pupila" (62\%), "emisión de hipótesis" (59\%), "representación mediante juego simbólico: oculistas" (29\%), "experimentación: contracción y dilatación de la pupila" (24\%), "utilización de instrumento: linterna" (15\%), "identificación de ideas en material audiovisual" (12\%) e "identificación de ideas en material escrito" (12\%). Y de los actitudinales: "curiosidad ante fenómenos cotidianos" (56\%), "creatividad en la emisión de hipótesis" (41\%), "fomento del trabajo en equipo y espiritu cooperativo" (24\%), "interés y disfrute por las ciencias" (18\%) y "autonomía en el manejo de una linterna" (12\%).

Tras analizar los resultados del examen final, pensamos que, en líneas generales, se obtuvieron unos valores satisfactorios si tenemos en cuenta los déficits -científicos y didácticoscon los que llegaron a la asignatura EACN1.

¿Se han producido progresos estadísticamente significativos en dichos conocimientos?

La categorización de las respuestas de $\mathrm{CC}$ y $\mathrm{CD}$ se recoge en las Tablas 7 y 8, respectivamente.

TABLA 7. Categorización del CC del alumnado en el postest

\begin{tabular}{ccc}
\hline $\begin{array}{c}\text { CATEGORÍA } \\
\text { (CC) }\end{array}$ & $\begin{array}{c}\text { TASA DE } \\
\text { ACIERTO }\end{array}$ & ALUMNAS \\
\hline Muy adecuado & Más del $90 \%$ & A1, A6, A9, A12, A13, A15, A17, A18, A22, A29, A30 \\
\hline Bastante adecuado & Entre $80-90 \%$ & A5, A8, A14, A16, A21, A27, A32, A33 \\
\hline
\end{tabular}




\begin{tabular}{ccc}
\hline $\begin{array}{c}\text { CATEGORÍA } \\
(\text { CC) }\end{array}$ & $\begin{array}{c}\text { TASA DE } \\
\text { ACIERTO }\end{array}$ & ALUMNAS \\
\hline Adecuado & Entre $70-79 \%$ & A3, A4, A11, A20, A23, A24, A25, A28 \\
\hline Aceptable & Entre $60-69 \%$ & A7, A10, A19, A31, A34 \\
\hline Poco aceptable & Entre $50-59 \%$ & A26 \\
\hline Nada aceptable & Menos del $50 \%$ & A2 \\
\hline
\end{tabular}

Fuente: elaboración propia

TABLA 8. Categorización del CD del alumnado en el postest

\begin{tabular}{ccc}
\hline $\begin{array}{c}\text { CATEGORÍA } \\
\text { (CD) }\end{array}$ & $\begin{array}{c}\text { TASA DE } \\
\text { ACIERTO }\end{array}$ & ALUMNAS \\
\hline Muy adecuado & Más de1 90\% & A7, A12, A13, A15, A18, A19, A22 \\
\hline Bastante adecuado & Entre $80-90 \%$ & A1, A6, A9, A11, A14, A20, A25, A32, A33 \\
\hline Adecuado & Entre 70-79\% & - \\
\hline Aceptable & Entre 60-69\% & A5, A23, A24, A27, A29, A34 \\
\hline Poco aceptable & Entre 50-59\% & - \\
\hline Nada aceptable & Menos del 50\% & A2, A3, A4, A8, A10, A16, A17, A21, A26, A28, A30, \\
& & A31 \\
\hline
\end{tabular}

Fuente: elaboración propia

Si contrastamos los resultados obtenidos entre pretest y postest, se observa una mejoría en las estudiantes -más en CC que en CD- tras cursar EACN1. Para estudiar si estas diferencias eran estadísticamente aplicamos la prueba de Wilcoxon y se obtuvo:

$$
\begin{aligned}
& Z=-5,09(p<0,00) \text {; entre CC (pretest) y CC (postest) } \\
& Z=-4,86(p<0,00) \text {; entre CD (pretest) y CD (postest) }
\end{aligned}
$$

Por tanto, existen diferencias estadísticamente significativas en favor del postest -en CC y CD- sobre el "cuerpo humano y la salud", tras aplicar nuestra propuesta.

Por último, calculamos el $\rho$ de Spearman entre CC (postest) y CD (postest) y obtuvimos que hay una relación significativa estadísticamente $(p=0,02)$ entre ambas variables.

\section{CONCLUSIONES}

Nuestro trabajo se sitúa en el ámbito de la formación inicial de maestras; en particular, a la enseñanza del "cuerpo humano y hábitos saludables", que se abordó en la asignatura "Enseñanza y Aprendizaje de las Ciencias de la Naturaleza I", impartida por el área de DCE, en el Grado de EI.

Desdoblamos el interrogante central -efectos de una propuesta formativa- en dos problemas principales; sólo nos ocupamos de los conocimientos científicos (CC) y didácticos (CD).

En relación con PP1, podemos decir que los conocimientos iniciales -científicos y didácticosde nuestras estudiantes tenían carencias importantes, aunque también logros que podíamos aprovechar. Pero, quizás, los hallazgos más interesantes se detectaron en la búsqueda de las razones:

- Respecto a los CC (pretest), podríamos acudir al consabido "no han cursado un Bachillerato de Ciencias" como causa de la situación. Sin embargo, los resultados de nuestra 
experiencia apuntan a otros factores. No disponemos de datos concluyentes, pero, quizás, cursar una modalidad de Bachillerato no implica saber trasferir los aprendizajes a hechos, fenómenos y acontecimientos cotidianos. Sin dicha trasferencia, sólo serán aprendizajes académicos.

-Respecto a los CD (pretest), tampoco se puede acudir al "olvido". No quisiéramos entrar en las otras materias, pero hemos constatado que nuestras futuras maestras no han sido capaces de utilizar los conocimientos pedagógicos adquiridos anteriormente. Nos parece obligado -cada vez más- reflexionar colectivamente de qué competencias profesionales queremos desarrollar y a qué nivel en la formación inicial.

En relación al PP2, podemos decir que es posible -y, en base a nuestra experiencia, deseableintegrar la formación científica y didáctica. Este enfoque da más respuestas a lo que necesita profesionalmente una maestra.

Además, hemos constatado avances importantes en CC (postest) y en CD (postest) y una alta relación entre ellos. Esto pone de manifiesto no sólo la eficacia de la propuesta, sino que el aprendizaje de ambos conocimientos se produce "conjuntamente". Aprender a enseñar ciencias implica también aprender ciencias. Esta afirmación debería hacer reflexionar a los que se guían por otras creencias.

\section{Referencias}

Acevedo, J.A. (2010). Formación del Profesorado de Ciencias y Enseñanza de la Naturaleza de la Ciencia. Revista Eureka sobre Enseñanza y Divulgación de las Ciencias, 7(3), 653-660. DOI: 10.25267/Rev_Eureka_ensen_divulg_cienc.2010.v7.i3.04

Aragüés, A. y Sáez, M.J. (2014). Análisis de una experiencia con maestros en formación: modelo de reacción química y ósmosis. En M.A. Heras (coord.), XXVI Encuentros de Didáctica de las Ciencias Experimentales (pp. 277-284). Huelva: Serv. Publicaciones Universidad.

Archila, P.A. (2012). La investigación en argumentación y sus implicaciones en la formación inicial de profesores de ciencias. Revista Eureka sobre Enseñanza y Divulgación de las Ciencias, 9(3), 361-375. DOI: 10498/14864

Arias, A., Álvarez, M. y Álvarez, F.J. (2013). Concepciones del profesorado en FI sobre los roles de docentes y discentes en el aprendizaje de las ciencias en EI y EP. Enseñanza de las Ciencias, núm. extra, 194-201. Recuperado de https://ddd.uab.cat/record/175085

Bargiela, I.M., Puig, B. y Blanco, P. (2018). Las prácticas científicas en infantil. Una aproximación al análisis del currículum y planes de formación del profesorado de Galicia. Enseñanza de las Ciencias, 36(1), 7-23. DOI: 10.5565/rev/ensciencias.2311

Bravo, J.L. y Cañada, F. (2016). ¿Saben realmente lo que dicen los futuros maestros antes de recibir instrucción en ciencias? En J.L. Bravo (ed.), XXVII Encuentro de Didáctica de las Ciencias Experimentales (pp. 1541-1548). Badajoz: UEX-ÁPICE.

Calafell, G. y Banqué, N. (2017). La Feria de Ciencias, una oportunidad para ambientalizar una asignatura de la formación inicial de maestros en educación infantil. Enseñanza de las Ciencias, núm. extra, 3117-3124. Recuperado de https://ddd.uab.cat/record/184010

Cantó, J., Pro, A. y Solbes, J. (2016). ¿Qué ciencias se enseñan y cómo se hace en las aulas de educación infantil? La visión de los maestros en formación inicial. Enseñanza de las Ciencias, 34(3), 25-50. DOI: 10.5565/rev/ensciencias. 1870

Cantó, J., Pro, A. y Solbes, J. (2017). ¿Qué resultados de aprendizaje alcanzan los futuros maestros de Infantil cuando planifican unidades didácticas de ciencias? Revista Eureka sobre Enseñanza y Divulgación de las Ciencias, 14(3), 666-688. DOI: 10498/19515

Cantó, J. y Solbes, J. (2014). ¿Qué les interesa a los futuros maestros de infantil de la Ciencia? En M. A. Heras (coord.), XXVI Encuentros de Didáctica de las Ciencias Experimentales (pp. 852-857). Huelva: Serv. Publicaciones Universidad. 
Díaz, N. (2017). ¿Cómo trabajar indagación en el aula de infantil? Análisis de las debilidades y fortalezas de los maestros en formación. Enseñanza de las ciencias, núm. extra, 1965-1970. Recuperado de https://ddd.uab.cat/record/184313

Do Carmo, C.M., Jiménez-Liso, M.R., López, F., Porlán, R. y Rivero, A. (2016). La práctica de la enseñanza de las ciencias: estrategias para el cambio. En J.L. Bravo (ed.), XXVII Encuentro de Didáctica de las Ciencias Experimentales (pp. 1607-1616). Badajoz: UEX-ÁPICE.

Eugenio, M., Aragón, L., Jiménez-Tenorio, N. y Vicente, J.J. (2016). Análisis de las creencias pedagógicas y científicas de futuros maestros de infantil antes y después de una propuesta didáctica en torno a la alfabetización científica. En J.L. Bravo (ed.), XXVII Encuentro de Didáctica de las Ciencias Experimentales (pp. 1197-1204). Badajoz: UEX-ÁPICE.

Ferrer, L.M., De Echave, A. y Mateo, E. (2016). Análisis de Trabajos Prácticos en laboratorio e implicaciones para la asignatura Ciencias de la Naturaleza del Grado de Maestro de Educación Infantil. En J.L. Bravo (ed.), XXVII Encuentro de Didáctica de las Ciencias Experimentales (pp. 219-226). Badajoz: UEX-ÁPICE.

Jiménez, R. (2012). Ayer, hoy y mañana de la investigación en la enseñanza de las ciencias. En J.M. Domínguez (ed.), XXV Encuentro de Didácticas de las Ciencias Experimentales (pp. 2145). Santiago Compostela: USC-ÁPICE.

López-Banet, L. y Pro, A. (2016). Valoración de la enseñanza de las ciencias desde la perspectiva de los estudiantes del Grado de Educación Infantil. En J.L. Bravo (ed.), XXVII Encuentro de Didáctica de las Ciencias Experimentales (pp. 271-278). Badajoz: UEX-ÁPICE.

López-Luengo, M.A., Gil, M., Verde, A. y Vallés, C. (2012). Análisis de una propuesta de formación científica en el grado de Maestro de EI. En J.M. Domínguez (ed.), XXV Encuentro de Didácticas de las Ciencias Experimentales (pp. 1141-1148). Santiago Compostela: USCÁPICE.

López-Luengo, M.A., Vallés, C. y Gil, C. (2016). Análisis de una propuesta de formación científica en el grado de Educación Infantil. En J.L. Bravo (ed.), XXVII Encuentro de Didáctica de las Ciencias Experimentales (pp. 73-80). Badajoz: UEX-ÁPICE.

Lorca, A., Vázquez, B. y Rosa, S. (2014). Los videojuegos para el profesorado en formación inicial en EI en la enseñanza de las Ciencias de la Naturaleza. En M.A. Heras (coord.), XXVI Encuentros de Didáctica de las Ciencias Experimentales (pp. 781-788). Huelva: Serv. Publicaciones Universidad.

Marín, N. (2014). Enseñanza de las ciencias desde el punto de vista del constructivismo orgánico. Enseñanza de las Ciencias, 32(2), 221-237. DOI: 10.5565/rev/ensciencias.775

Mazas, B. y Bravo, B. (2016). Dificultades de los maestros en formación asociadas a la transposición didáctica en Educación Infantil. En J.L. Bravo (ed.), XXVII Encuentro de Didáctica de las Ciencias Experimentales (pp. 319-326). Badajoz: UEX-ÁPICE.

Moreno, C., González, S. y Meneses, J.A. (2017). Enseñanza de las ciencias a través de la metodología indagatoria en educación infantil. Proyecto limpiemos el agua. Enseñanza de las Ciencias, núm. extra, 989-994. Recuperado de https://ddd.uab.cat/record/184517

Pablos, M. y Verde, A. (2013). Influencia de la procedencia de los alumnos en los resultados obtenidos en DCE (Grado de EI). Enseñanza de las Ciencias, núm. extra, 2632-2638. Recuperado de https://ddd.uab.cat/record/175502

Pro Bueno, A. (2016). ¿Qué problemas tiene la formación inicial de maestros en España? En J.L. Bravo (ed.), XXVII Encuentro de Didáctica de las Ciencias Experimentales (pp. 1619-1625). Badajoz: UEX-ÁPICE.

Pro Chereguini, C. (2017a). Conocimiento didáctico de los maestros de Educación Infantil: ¿cómo evalúan hábitos saludables del alumnado? En J.J. Maquilón (ed.), Investigaciones sobre la formación docente en el siglo XXI (pp. 173-185). Murcia: Serv. Public. Universidad. 
Pro Chereguini, C. (2017b). ¿Cómo plantean los futuros maestros una actividad experimental para Educación Infantil? Actas $V$ Congreso Internacional de Investigación e Innovación en Educación Infantil y Primaria (s.p.). Murcia: Serv. Public. Universidad.

Real Decreto 1630/2006, de 29 de diciembre, por el que se establecen las enseñanzas mínimas del segundo ciclo de Educación Infantil. (BOE, 4, 474-482).

Romero-López, M.C., Jiménez-Tejada, M.P., González-García, F., Carrillo-Rosúa, F.J., Barón, S.D., Casas-Castillo, R. y Ruiz-Rodríguez, L. (2016). Educación Nutricional mediante trabajo colaborativo multidisciplinar con un enfoque Aprendizaje-Servicio. En J.L. Bravo (ed.), XXVII Encuentro de Didáctica de las Ciencias Experimentales (pp. 1187-1194). Badajoz: UEX-ÁPICE.

Sánchez, J., Conde, M.C. y Garrido, A. (2014). Análisis de los contenidos de educación para el consumo alimentario propuestos por estudiantes del Grado de Educación Infantil. En M.A. Heras (coord.), XXVI Encuentros de Didáctica de las Ciencias Experimentales (pp. 634-642). Huelva: Serv. Publicaciones Universidad.

Sanz, J. y Gutiérrez, L. (2016). Talleres científicos con niños y niñas en el Grado de Educación Infantil: un modelo de investigación-acción y una iniciativa Universidad-Escuela. En J.L. Bravo (ed.), XXVII Encuentro de Didáctica de las Ciencias Experimentales (pp. 141-148). Badajoz: UEX-ÁPICE.

Sanz, J. y Gutiérrez, L. (2017). Talleres de ciencias en grado de infantil: bases de una práctica reflexiva para el profesorado en formación. Enseñanza de las Ciencias, núm. extra, 20092014. Recuperado de https://ddd.uab.cat/record/184307

Solé-Llussà, A., Aguilar, D., Ibáñez, M. y Coiduras, J.L. (2017). Análisis de la indagación científica a partir de las comunicaciones realizadas en congresos de ciencias dirigidos a alumnos de Educación Infantil y Primaria. Enseñanza de las Ciencias, núm. extra, 1103-1108. Recuperado de https://ddd.uab.cat/record/184501

Solé-Llussà, A., Aguilar, D., Ibáñez, M. y Coiduras, J.L. (2018). Análisis de la comunicación de experiencias indagadoras presentadas en congresos de ciencia dirigidos a alumnos de educación infantil y primaria. Revista Eureka sobre Enseñanza y Divulgación de las Ciencias, 15(1), 1302. DOI: 10.25267/Rev_Eureka_ensen_divulg_cienc.2018.v15.i1.1302

Valín, A., Moledo, L., López Maceiras, M. y García-Rodeja, I. (2012). Un proyecto de ciencias en el aula de Infantil: Las abejas. En J.M. Domínguez (ed.), XXV Encuentro de Didácticas de las Ciencias Experimentales (pp. 787-794). Santiago Compostela: USC-APICE.

Vestena, R.D.F., Pretto, V. y Marschall, J. (2017). As Ciências da Natureza na Educação Infantil a partir de uma proposta de Estágio Supervisionado. Enseñanza de las ciencias, núm. extra, 2201-2206. Recuperado de https://ddd.uab.cat/record/184276

\section{CÓMO CITAR ESTE ARTÍCULO}

Pro Chereguini, C., Inglés Carrillo, C. y Gómez Mármol, A. (2020). ¿Qué aprenden unos estudiantes en una experiencia formativa de Didáctica de las Ciencias Experimentales en el Grado de Educación Infantil? Didáctica de las ciencias experimentales y sociales, 38, 97-114. DOI: $10.7203 /$ DCES.38.16174. 


\section{Anexo}

ANEXO 1. Descripción propuesta "Cómo enseñar el cuerpo humano y los hábitos saludables en EI"

\begin{tabular}{|c|c|c|}
\hline Sección & $\begin{array}{c}\text { Interrogante } \\
\text { principal }\end{array}$ & Descripción \\
\hline \multirow[t]{4}{*}{1} & \multirow{4}{*}{$\begin{array}{l}\text { ¿Qué dice el } \\
\text { currículum de } \\
\text { EI de la } \\
\text { enseñanza de } \\
\text { las ciencias? }\end{array}$} & $\begin{array}{l}\text { - Explicación del currículum de EI: objetivos de etapa, estructura, bloques de } \\
\text { contenidos y criterios de evaluación }\end{array}$ \\
\hline & & - Lectura: Documento 1 de EACN1 \\
\hline & & - Debate: Presencia de las Ciencias en las aulas en las Prácticas de Enseñanza \\
\hline & & $\begin{array}{l}\text { - Trabajo individual: Presencia de las Ciencias en el currículum oficial: cuerpo } \\
\text { humano y salud }\end{array}$ \\
\hline \multirow[t]{7}{*}{2} & \multirow{7}{*}{$\begin{array}{l}\text { ¿Qué Ciencias } \\
\text { podemos } \\
\text { enseñar a un } \\
\text { niño de EI? }\end{array}$} & $\begin{array}{l}\text { - Explicación sobre qué son las Ciencias, qué tipos de conocimientos abordan, } \\
\text { cómo evoluciona, qué repercusiones tiene en nuestra vida cotidiana... }\end{array}$ \\
\hline & & - Lectura: Documento 2 de EACN1 \\
\hline & & $\begin{array}{l}\text { - Debate: Necesidades de un niño de 0-6 que se pueden atender desde las Ciencias } \\
\text { (no qué Ciencias pueden ser útiles a un niño de EI) }\end{array}$ \\
\hline & & $\begin{array}{l}\text { - Trabajo grupal fuera del aula: Realización de un vídeo y una hoja de trabajo } \\
\text { para enseñar a un niño qué es un científico }\end{array}$ \\
\hline & & $\begin{array}{l}\text { - Trabajo grupal: Análisis para trabajar el cuerpo humano y la salud: contenidos, } \\
\text { actividades y recursos }\end{array}$ \\
\hline & & - Debate: Puesta en común de los trabajos realizados \\
\hline & & $\begin{array}{l}\text { - Trabajo grupal: Identificación de contenidos por ciclos sobre el cuerpo humano } \\
\text { y la salud en EI }\end{array}$ \\
\hline \multirow[t]{4}{*}{3} & \multirow{4}{*}{$\begin{array}{l}\text { ¿Qué logros y } \\
\text { dificultades } \\
\text { tiene el } \\
\text { alumnado para } \\
\text { aprenderlo? }\end{array}$} & $\begin{array}{l}\text { - Explicación de logros y dificultades del alumnado de EI para aprender Ciencias. } \\
\text { - Lectura: Documento } 3 \text { de EACN1 }\end{array}$ \\
\hline & & $\begin{array}{l}\text { - Debate: Consecuencias de las características psico-socio-cognitivas del niño de } \\
\text { EI para aprender Ciencias; en particular, cuerpo humano y salud }\end{array}$ \\
\hline & & $\begin{array}{l}\text { - Trabajo grupal: Diseñar una entrevista para conocer los hábitos del alumnado de } \\
\text { EI sobre un tópico relacionado con la salud }\end{array}$ \\
\hline & & $\begin{array}{l}\text { - Trabajo grupal fuera del aula: Aplicar la entrevista a niños de EI de 5-6 años } \\
\text { (dos por miembro del grupo) }\end{array}$ \\
\hline \multirow[t]{4}{*}{4} & \multirow{4}{*}{$\begin{array}{l}\text { ¿Qué } \\
\text { secuencia } \\
\text { didáctica } \\
\text { podemos } \\
\text { utilizar? }\end{array}$} & $\begin{array}{l}\text { - Explicación de secuencias: secuencia tradicional; secuencia introducción- } \\
\text { construcción-aplicación-revisión; secuencia de trabajo por proyectos }\end{array}$ \\
\hline & & - Lectura: Documento 4 de EACN1 \\
\hline & & - Debate: Trasposición didáctica en EI de la metodología científica \\
\hline & & $\begin{array}{l}\text { - Trabajo grupal: Diseñar una actividad experimental para enseñar Ciencias en EI, } \\
\text { siguiendo la metodología científica }\end{array}$ \\
\hline \multirow[t]{4}{*}{5} & \multirow[t]{4}{*}{$\begin{array}{l}\text { ¿Qué } \\
\text { actividades } \\
\text { podemos } \\
\text { realizar? }\end{array}$} & $\begin{array}{l}\text { - Explicación de orientaciones metodológicas del currículum; elementos a } \\
\text { considerar en el diseño de actividades (intención, contenidos, roles, recursos...). } \\
\text { - Lectura: Documento } 5 \text { de EACN1 }\end{array}$ \\
\hline & & $\begin{array}{l}\text { - Trabajo individual: Análisis de actividades para trabajar el cuerpo humano y } \\
\text { creación de hábitos saludables: contenidos e intenciones educativas. }\end{array}$ \\
\hline & & $\begin{array}{l}\text { - Visualizar y reflexionar sobre el vídeo "La boca y los dientes" de la serie "Érase } \\
\text { una vez la vida" (https://www.youtube.com/watch?v=6dxr9RAndKM) }\end{array}$ \\
\hline & & $\begin{array}{l}\text { - Trabajo grupal fuera del aula: Diseño y exposición de una secuencia de } \\
\text { actividades para EI sobre un tema del cuerpo humano o la salud }\end{array}$ \\
\hline \multirow[t]{3}{*}{6} & \multirow{3}{*}{$\begin{array}{l}\text { ¿Cómo } \\
\text { evaluamos el } \\
\text { aprendizaje } \\
\text { del alumnado? }\end{array}$} & - Explicación qué es evaluar: el qué, el cómo y para qué \\
\hline & & - Lectura: Documento 6 de EACN1 \\
\hline & & - Debate: Puesta en común de resultados de las entrevistas a alumnos de EI \\
\hline
\end{tabular}


